\title{
Nonzero coefficients in restrictions and tensor products of supercharacters of $U_{n}(q)$ (extended abstract)
}

\author{
Stephen Lewis ${ }^{1}$ and Nathaniel Thiem ${ }^{2}$ \\ ${ }^{1}$ Department of Mathematics, University of Washington \\ ${ }^{2}$ Department of Mathematics, University of Colorado at Boulder
}

\begin{abstract}
The standard supercharacter theory of the finite unipotent upper-triangular matrices $U_{n}(q)$ gives rise to a beautiful combinatorics based on set partitions. As with the representation theory of the symmetric group, embeddings of $U_{m}(q) \subseteq U_{n}(q)$ for $m \leq n$ lead to branching rules. Diaconis and Isaacs established that the restriction of a supercharacter of $U_{n}(q)$ is a nonnegative integer linear combination of supercharacters of $U_{m}(q)$ (in fact, it is polynomial in $q$ ). In a first step towards understanding the combinatorics of coefficients in the branching rules of the supercharacters of $U_{n}(q)$, this paper characterizes when a given coefficient is nonzero in the restriction of a supercharacter and the tensor product of two supercharacters. These conditions are given uniformly in terms of complete matchings in bipartite graphs.
\end{abstract}

Résumé. La théorie standard des supercaractères des matrices triangulaires supérieures unipotentes finies $U_{n}(q)$ donne lieu à une merveilleuse combinatoire basée sur les partitions d'ensembles. Comme avec la théorie des représentations du groupe symétrique, Les plongements $U_{m}(q) \subseteq U_{n}(q)$ pour $m \leq n$ mènent aux règles de branchement. Diaconis et Isaacs ont montré que la restriction d'un supercaractère de $U_{n}(q)$ est une combinaison linéaire des supercaractères de $U_{m}(q)$ avec des coefficients entiers non négatifs (en fait, elle est polynomiale en $q$ ). Dans une première étape vers la compréhension de la combinatoire des coefficients dans les règles de branchement des supercaractères de $U_{n}(q)$, ce texte caractérise les coefficients non nuls dans la restriction d'un supercaractère et dans le produit des tenseurs de deux supercaractères. Ces conditions sont données uniformément en termes des couplages complets dans des graphes bipartis.

Keywords: supercharacters, set-partitions, matching, bipartite graphs, unipotent upper-triangular matrices

\section{Introduction}

The representation theory of the finite groups of unipotent upper-triangular matrices $U_{n}\left(\mathbb{F}_{q}\right)$ has traditionally been a hard problem, where even enumerating the irreducible representations is a well-known wild problem. In fact, it is not even known if the number of irreducible representations is polynomial in $q$ (the Higman conjecture [12] suggests the affirmative). However, André [1, 2, 3, 4] and later Yan [18] demonstrated if one decomposes the regular representation into "nearly irreducible" pieces (called superrepresentations) rather than the usual irreducible pieces, one obtains a theory that is far more tractable with 
beautiful combinatorial underpinnings. Later, work of Arias-Castro, Diaconis and Stanley [7] demonstrated that this theory could even be used in place of the usual representation theory in an application to random walks, and for more general supercharacter theories, Otto [15] has shown that they can be used to bound nilpotence classes of nilpotent algebras.

While it has been a guiding principle that the supercharacter theory of $U_{n}(q)$ is analogous to the representation theory of the symmetric group $S_{n}$, many $U_{n}$-analogues of $S_{n}$ results remain to be worked out. Some of the known observations include

(a) The irreducible characters of $S_{n}$ are indexed by partitions of $n$, and the supercharacters of $U_{n}\left(\mathbb{F}_{q}\right)$ are indexed by (a $q$-analogue of) set partitions of $\{1,2, \ldots, n\}$ [1, 18, 7],

(b) For $S_{n}$, Young subgroups are a natural family of subgroups which give the corresponding character rings a Hopf structure through induction and restriction. Similarly, [16] defines an analogue to Young subgroups for $U_{n}(q)$, noting that while in the $S_{n}$-case the particular embedding of the subgroup typically does not matter, in the $U_{n}(q)$-case it is critical [14, 17]. These subgroups are indexed by set-partitions instead of integer partitions.

(c) As an algebra, the ring of symmetric functions model restriction/induction branching rules for the characters of $S_{n}$ considered simultaneously for all $n \geq 0$. The corresponding ring for the supercharacters of $U_{n}\left(\mathbb{F}_{q}\right)$ seems to be the ring of symmetric functions in non-commuting variables [16].

This paper attempts to better understand the combinatorics of branching rule coefficients for the supercharacters of $U_{n}\left(\mathbb{F}_{q}\right)$.

In the symmetric group case, the irreducible character $\chi^{\mu} \times \chi^{\nu}$ appears in the decomposition of the restricted character

$$
\operatorname{Res}_{S_{|\mu|} \times S_{|\nu|}}^{S_{|\lambda|}}\left(\chi^{\lambda}\right)
$$

only if $\mu, \nu \subset \lambda$. For $U_{n}(q)$ this paper gives both necessary and sufficient conditions for analogous result, using the close relationship between tensor products and restrictions in this case. In particular, the main results of this abstract are

Theorem 3.1. Given a set partition $\lambda$ and subgroup $U_{K} \subseteq U_{n}$, there is a bipartite graph $\Gamma_{K}(\lambda)$ such that the trivial character appears in the decomposition of $\operatorname{Res}_{U_{K}}^{U_{n}}\left(\chi^{\lambda}\right)$ if and only if the graph has a complete matching.

Theorem 4.1. Given set partitions $\lambda, \mu$, and $\nu$, there is a bipartite graph $\Gamma(\lambda, \mu, \nu)$ such that the $\chi^{\nu}$ appears in the decomposition of $\chi^{\lambda} \otimes \chi^{\mu}$ if and only if the graph has a complete matching.

Theorem 4.3. Given set partitions $\lambda, \mu$ and subgroup $U_{K} \subseteq U_{n}$, there is a bipartite graph $\Gamma_{K}(\lambda, \mu)$ such that the $\chi^{\mu}$ appears in the decomposition of $\operatorname{Res}_{U_{K}}^{U_{n}}\left(\chi^{\lambda}\right)$ if and only if the graph has a complete matching.

The bipartite graphs referenced in all three results have a uniform construction as described in Section 4.1. and are remarkably easy to construct given the initial data. However, the description of the bipartite graph in Theorem 3.1 is particularly nice, so we describe it separately in Section 3.1. A fundamental part of extending Theorem 3.1 to Theorem 4.1 and Theorem 4.3 is a result that rewrites tensor products as restriction, as follows. 
Theorem 4.2 Given two supercharacters $\chi^{\lambda}$ and $\chi^{\mu}$ of $U_{K}$, there exists a supercharacter $\chi^{\nu}$ and groups $U_{K^{\prime}} \subseteq U_{L}$ with $U_{K^{\prime}} \cong U_{K}$ such that $\chi^{\lambda} \otimes \chi^{\mu}$ (under the internal point-wise product) is the same character of $U_{K}$ as $q^{-r} \operatorname{Res}_{U_{K}^{\prime}}^{U_{L}}\left(\chi^{\nu}\right)$ is of $U_{K^{\prime}}$, where $r \in \mathbb{Z}_{\geq 0}$.

While these results do not give a complete description of the coefficients, [8] showed that these coefficients are always positive integers (in fact, polynomial in $q$ ). Thus, a more explicit understanding of the coefficients remains open.

The supercharacter theory studied in this paper is a particular example of a supercharacter theory that has a more general construction on algebra groups [8]. Generalizations of this approach have also been studied by André and Neto for maximal unipotents subgroups in other Lie types [5, 6]. This study of a particular supercharacter theory is somewhat different from recent work by [11], which attempts to find all the possible supercharacter theories for a given finite group.

\section{Acknowledgements}

Part of this work (in particular Theorem 3.1] was in Lewis' undergraduate honors thesis at the University of Colorado at Boulder. Thiem was supported in part by an NSF grant DMS-0854893.

\section{Preliminaries}

This section sets up the necessary combinatorics of set partitions, which differs from some of the more standard formulations. From this point of view, the parts of the set partition are less important than the relative sizes of the numbers in the same part. We then review the definition of a supercharacter theory, and recall the specific supercharacter theory of interest for the finite groups of unipotent upper-triangular matrices, as developed by André [1, 2, 3, 4] and Yan [18].

\subsection{Set partition combinatorics}

Fix a prime power $q$, and let $\mathbb{F}_{q}$ be the finite field with $q$ elements with additive group $\mathbb{F}_{q}^{+}$and multiplicative group $\mathbb{F}_{q}^{\times}$.

For a finite subset $K \subseteq \mathbb{Z}_{\geq 1}$, let

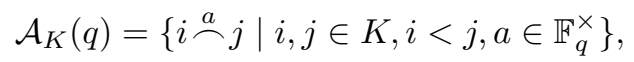

and

$$
\mathcal{A}(q)=\bigcup_{\substack{K \subseteq \mathbb{Z} \\|K|<\infty}} \mathcal{A}_{K}(q)
$$

where $\mathcal{A}_{\emptyset}(q)=\{\emptyset\}$. We will refer to the non-emptyset elements of $\mathcal{A}(q)$ as arcs.

Let

$$
\mathcal{M}(q)=\bigcup_{\substack{K \subseteq \mathbb{Z} \geq 1 \\|K|<\infty}} \mathcal{M}_{K}(q), \quad \text { where } \quad \mathcal{M}_{K}(q)=\left\{\text { finite multisets in } \mathcal{A}_{K}(q)\right\} .
$$

For $\lambda \in \mathcal{M}_{K}(q)$ and $j, k \in K$, let

$$
R_{j k}=\{j \stackrel{a}{\sim} k \in \lambda\}, \quad m_{j k}(\lambda)=\left|R_{j k}\right|, \quad \text { and } \quad \operatorname{wt}_{j k}(\lambda)=\sum_{j \stackrel{a}{a} k \in R_{j k}} a \in \mathbb{F}_{q},
$$


where $R_{j k}$ is a multiset. For example, if

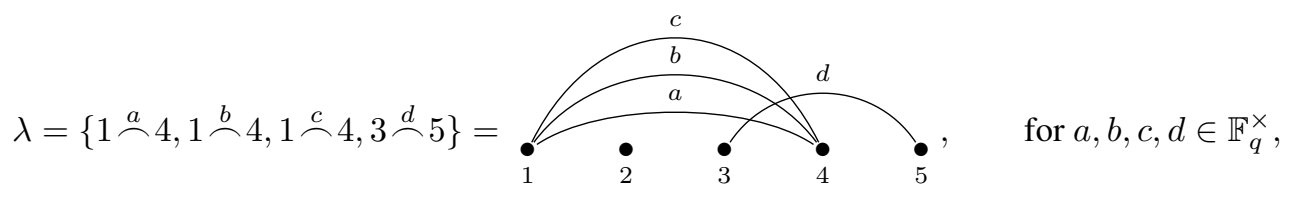

then

$$
R_{14}=\{1 \stackrel{a}{\lessgtr} 4,1 \stackrel{b}{\lessgtr} 4,1 \stackrel{c}{\subseteq} 4\}, \quad m_{14}(\lambda)=3, \quad \mathrm{wt}_{14}(\lambda)=a+b+c, \quad \text { and } \quad R_{23}=\emptyset .
$$

Note we will use a diagrammatic representation of multisets $\lambda \in \mathcal{M}_{K}(q)$, by associating to each element of $K$ a node (usually arranged along a horizontal line), and each $\operatorname{arc} i \stackrel{a}{\sim} j$ in $\lambda$ becomes a labeled edge connecting node $i$ to node $j$.

A $q$-set partition of $K$ is a multiset $\lambda \in \mathcal{M}_{K}(q)$ such that if $i \stackrel{a}{\sim} l, j \stackrel{b}{\triangle} k \in \lambda$ are two distinct arcs, then $i \neq j$ and $k \neq l$. Let

$$
\mathcal{S}(q)=\bigcup_{\substack{K \subseteq \mathbb{Z} \\|K|<\infty}} \mathcal{S}_{K}(q), \quad \text { where } \quad \mathcal{S}_{K}(q)=\left\{q \text {-set partitions in } \mathcal{M}_{K}(q)\right\}
$$

Note that 2-set partitions of $K$ are set partitions $\lambda$ of $K$ by the rule that $i$ and $j$ are in the same part if there is a sequence of $\operatorname{arcs} i \stackrel{1}{\sim} j_{1}, j_{1} \stackrel{1}{\sim} j_{2}, \ldots, j_{m-1} \stackrel{1}{\sim} j \in \lambda$. That is, the parts of the set partitions are the connected components of the diagrammatic representation of the 2 -set partition. For example,

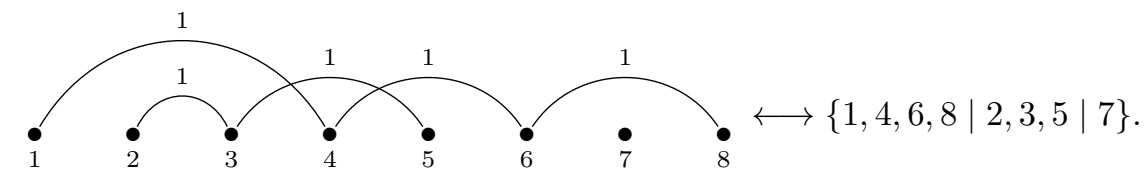

In this sense, $q$-set partitions are a $q$-analogue of set partitions (although, strictly speaking, they are $(q-1)$ analogues of set partitions).

Let $\lambda \in \mathcal{M}(q)$. A conflict in $\lambda$ over $K$ is either

(CL) A pair of distinct $\operatorname{arcs} i \stackrel{a}{\sim} l, j \stackrel{b}{\sim} k \in \lambda$ such that $i=j$ and $k<l$,

(CR) A pair of distinct $\operatorname{arcs} i \stackrel{a}{\sim} l, j \stackrel{b}{\sim} k \in \lambda$ such that $i<j$ and $k=l$,

(CB) A pair of distinct $\operatorname{arcs} i \stackrel{a}{\sim} l, j \stackrel{b}{\sim} k \in \lambda$ such that $i=j$ and $k=l$,

(CN) A nonempty multiset $\{i \stackrel{a}{\sim} k \in \lambda \mid i=j$ or $k=j\}$ for some $j \notin K$.

Example 1 The multiset

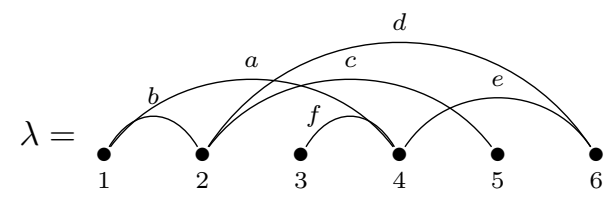


has conflicts

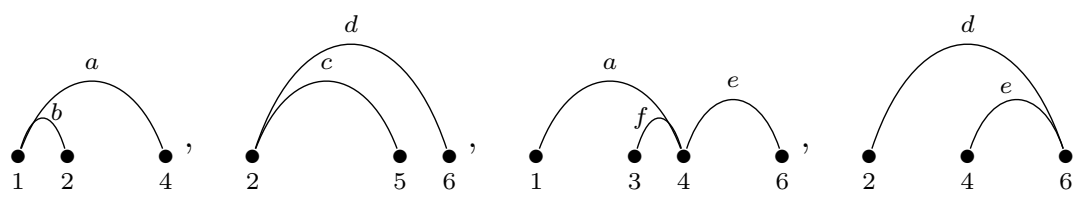

over $\{1,2,3,5,6\}$, where the conflicts are of type $(C L),(C L),(C N)$, and $(C R)$.

Conflicts are instances in a multiset which violate the conditions of membership in the set $\mathcal{S}_{K}(q)$.

Remark 1 There are a variety of q-analogues of set partition or Stirling numbers in the literature. This particular q-analogue of set partitions is different from the one introduced [10] and only seems to appear in connection with supercharacters. There is also a standard construction for q-Stirling numbers (see for example [9]), where the Stirling number $S(n, k ; q)$ is defined by " $q$-counting" the number of elements of $\mathcal{S}_{n}(2)$ with $n-k$ arcs. If we let $S_{q}(n, k)$ be the number of elements of $\mathcal{S}_{n}(q)$ with $n-k$ elements, we obtain a recursion

$$
\mathcal{S}_{q}(n, k)=\mathcal{S}_{q}(n-1, k-1)+k(q-1) \mathcal{S}_{q}(n-1, k),
$$

which is different from the recursion for $S(n, k ; q)$ in [9].

\subsection{Supercharacters of $U_{n}(q)$}

Supercharacters were first studied by André in relation to $U_{n}(q)$ as a way to find some more tractable way to understand the representation theory of $U_{n}(q)$. Diaconis and Isaacs [8] then generalized the concept to arbitrary finite groups, and we reproduce a version of this more general definition below.

A supercharacter theory of a finite group $G$ is a pair $(\mathcal{K}, \mathcal{X})$ where $\mathcal{K}$ is a partition of $G$ such that

(a) Each $K \in \mathcal{K}$ is a union of conjugacy classes,

(b) The identity element of $G$ is in its own part in $\mathcal{K}$,

and $\mathcal{X}$ is a set of characters of $G$ such that

(a) For each irreducible character $\psi$ of $G$ there is a unique $\chi \in \mathcal{X}$ such that $\langle\chi, \psi\rangle \neq 0$,

(b) The trivial character $\mathbb{1} \in \mathcal{X}$,

(c) The characters of $\mathcal{X}$ are constant on the parts of $\mathcal{K}$,

(d) $|\mathcal{K}|=|\mathcal{X}|$.

We will refer to the parts of $\mathcal{K}$ as superclasses and the characters of $\mathcal{X}$ as supercharacters. For more information on the implications of these axioms see [8] (including some redundancies in the definition).

For $n \in \mathbb{Z}_{\geq 1}$, let $M_{n}\left(\mathbb{F}_{q}\right)$ be the ring of $n \times n$ matrices with entries in the finite field $\mathbb{F}_{q}$ with $q$ elements. Let

$$
U_{n}(q)=\left\{u \in M_{n}\left(\mathbb{F}_{q}\right) \mid u_{j i}=0, u_{i i}=1, u_{i j} \in \mathbb{F}_{q}, i<j\right\}
$$

be the group of unipotent upper-triangular matrices. For $K \subseteq\{1,2, \ldots, n\}$, let

$$
U_{K}(q)=\left\{u \in U_{n}(q) \mid u_{i j}=0, i<j \text {, unless } i, j \in K\right\} .
$$


Note that $U_{K}(q) \cong U_{|K|}(q)$.

For $U_{K}(q)$ there is a standard example of a supercharacter theory developed by André and Yan, where $\mathcal{K}$ and $\mathcal{X}$ are indexed by $q$-set partitions of $K$. For the purpose of this paper it suffices to recall the definition of the supercharacters. Fix a nontrivial group homomorphism,

$$
\vartheta: \mathbb{F}_{q}^{+} \longrightarrow \mathbb{C}^{\times} \text {. }
$$

For $\lambda \in \mathcal{S}_{K}(q)$, there is a supercharacter $\chi^{\lambda}$ given by

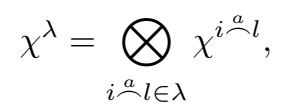

where each $\chi^{i \stackrel{a}{\sim} l}$ is an irreducible character of $U_{K}(q)$ whose value on the superclass indexed by $\mu \in$ $\mathcal{S}_{K}(q)$ is

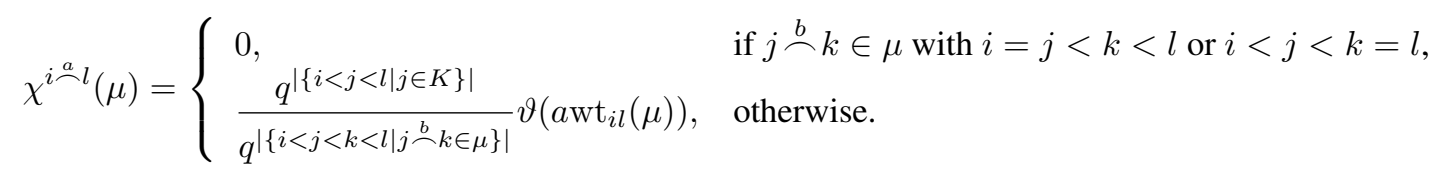

It can be quickly verified that the linear supercharacters of $U_{K}(q)$ correspond to $\lambda \in \mathcal{S}_{K}(q)$ with $i \stackrel{a}{\sim} l \in \lambda$ implies $\{i<j<l \mid j \in K\}=\emptyset$; the trivial character is $\chi^{\emptyset}$.

The superclass $\{1\}$ is indexed by $\emptyset \in \mathcal{S}_{L}(q)$. Thus, for $\lambda \in \mathcal{M}_{L}(q)$ with $L \subseteq \mathbb{Z}_{\geq 1}$, the degree of $\chi^{\lambda}$ is

$$
\chi^{\lambda}(1)=\prod_{i \underset{a}{a} l \in \lambda} q^{|\{i<j<l \mid j \in L\}|} .
$$

If $K \subseteq L$, then define

$$
r_{K}^{L}(\lambda)=|\{(j, i \stackrel{a}{=} l) \in L \times \lambda \mid i<j<l, j \notin K\}| .
$$

Note that if $\lambda \in \mathcal{M}_{L}(q) \cap \mathcal{M}_{K}(q)$ then $q^{r_{K}^{L}(\lambda)}$ is the ratio of the degrees of $\chi^{\lambda}$ as a character of $U_{L}(q)$ and $\chi^{\lambda}$ as a character of $U_{K}(q)$. It therefore is a constant that frequently comes up in the restriction of supercharacters. In fact, by inspection, if $\lambda \in \mathcal{M}_{L}(q) \cap \mathcal{M}_{K}(q)$, then

$$
\operatorname{Res}_{U_{K}(q)}^{U_{L}(q)}\left(\chi^{\lambda}\right)=q^{r_{K}^{L}(q)} \chi^{\lambda}
$$

In general, supercharacters are orthogonal with respect to the usual inner product on class functions, and for $\lambda, \mu \in \mathcal{S}_{K}(q)$,

$$
\left\langle\chi^{\lambda}, \chi^{\mu}\right\rangle=\delta_{\lambda \mu} q^{|\mathcal{C}(\lambda)|}, \quad \text { where } \mathcal{C}(\lambda)=\{(i \stackrel{a}{\sim} k, j \stackrel{b}{\circ} l) \in \lambda \times \lambda \mid i<j<k<l\},
$$

is the set of crossings in $\lambda$.

The papers [16, 17, 18] describe local rules for computing restrictions and tensor products. In principle, therefore, one can easily compute restrictions and tensor products in a recursive, algorithmic fashion (see [16] for a detailed description of this algorithm, and [13] for an implementation of this algorithm in Python). However, this algorithm does not give an obvious combinatorial interpretation of the resulting coefficients. 


\section{Coefficient of trivial character}

This section investigates the coefficient of the trivial character $\mathbb{1}=\chi^{\emptyset}$ in the restriction from $U_{L}(q)$ to a subgroup $U_{K}(q)$. In particular, Theorem 3.1 characterizes when the coefficient of $\mathbb{1}$ is nonzero in the restriction of a supercharacter. Although the theorem seems somewhat specific, in later sections we will use it to analyze the coefficients of arbitrary supercharacters in both restrictions and tensor products.

\subsection{Main result}

Given a set partition $\lambda \in \mathcal{S}_{L}(q)$ and a subset $K \subseteq L$, let $\Gamma_{K}(\lambda)$ be the bipartite graph given by vertices

$$
\begin{aligned}
& V_{\bullet}=\{i \frown j \in \lambda \mid i, j \in K\} \\
& V_{\circ}=\{i \frown j \in \lambda \mid i, j \notin K\},
\end{aligned}
$$

and an edge from $j \frown k \in V_{\bullet}$ to $i \frown l \in V_{\circ}$ if $i<j<k<l$. Note that this graph has in general far fewer vertices than $\lambda$ has arcs. The following theorem is the main result of the paper, and is a model for the remaining results in this paper.

Theorem 3.1 Let $K \subseteq L \subseteq \mathbb{Z}_{\geq 1}$ be finite sets, and let $\lambda \in \mathcal{S}_{L}(q)$ be a q-set partition. Then

$$
\left\langle\operatorname{Res}_{U_{K}}^{U_{L}}\left(\chi^{\lambda}\right), \mathbb{1}\right\rangle \neq 0
$$

if and only if the graph $\Gamma_{K}(\lambda)$ has a complete matching from $V_{\bullet}$ to $V_{\circ}$.

Remark 2 The complete matching referred to in Theorem 3.1 is a one-sided matching. That is, every element in $V_{\bullet}$ must be matched to a corresponding element in $V_{0}$, but there could potentially be elements of $V_{\circ}$ not matched to elements of $V_{\bullet}$. For example, if
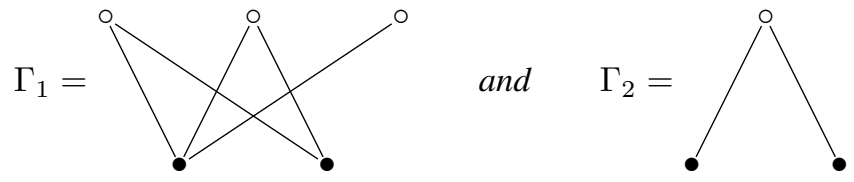

then $\Gamma_{1}$ has a complete matching from $V_{\bullet}$ to $V_{\circ}$ and $\Gamma_{2}$ does not.

Example 2 If $K=\{1,4,5,6,7,9\}$ and

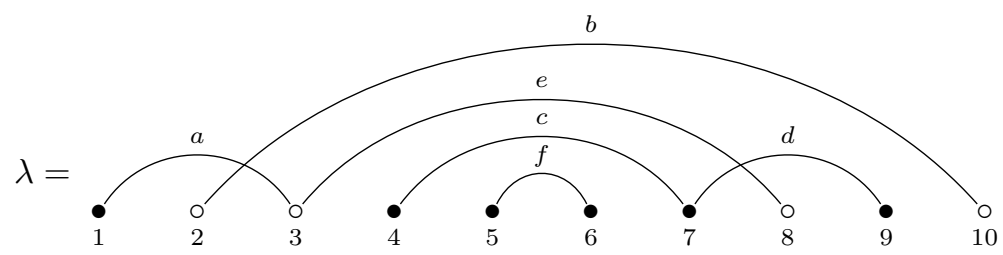

then $V_{\bullet}=\{4 \stackrel{c}{\subseteq} 7,5 \stackrel{f}{\subseteq} 6,7 \stackrel{d}{\subseteq} 9\}, V_{\circ}=\{2 \stackrel{b}{\frown} 10,3 \stackrel{e}{\subseteq} 8\}$, and

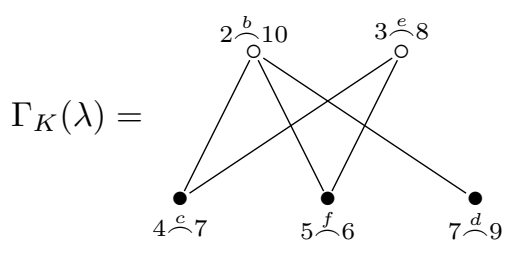


Since this graph has no complete matchings from $V_{\bullet}$ to $V_{\circ}$, by Theorem $3.1\left\langle\operatorname{Res}_{U_{K}}^{U_{L}}\left(\chi^{\lambda}\right), \mathbb{1}\right\rangle=0$.

\section{Tensor products and general restriction coefficients}

Theorem 3.1 in fact is sufficiently strong that analogous statements can be made for the coefficients of arbitrary supercharacters in the decomposition of tensor products and restrictions. This section begins by developing the appropriate generalization to the graph $\Gamma_{K}(\lambda)$. We then state Theorem 4.1 for tensor products and Theorem 4.3 for restrictions. Along the way, Theorem 4.2 describes how characters corresponding to multisets are the same as restrictions from certain set partitions (up to a scalar multiple).

\subsection{A generalized bipartite graph}

Given $\lambda \in \mathcal{M}(q)$, perturb the arcs such that they stack on top of one-another in the following fashion.

(TL) If $i \stackrel{a}{\sim} j, i \stackrel{b}{\sim} k \in \lambda$ with $j<k$, then the left endpoint of $i \stackrel{b}{\sim} k$ is above the left endpoint of $i \stackrel{a}{\sim} j$,

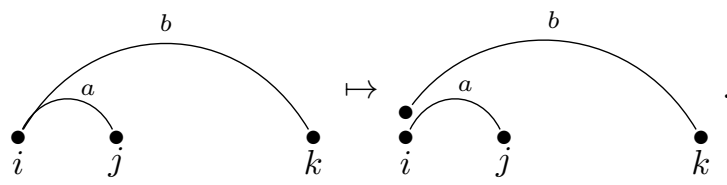

(TR) If $i \stackrel{a}{\sim} k, j \stackrel{b}{\sim} k \in \lambda$ with $i<j$, then the right endpoint of $i \stackrel{a}{\sim} k$ is above the right endpoint of $j \stackrel{b}{\sim} k$,

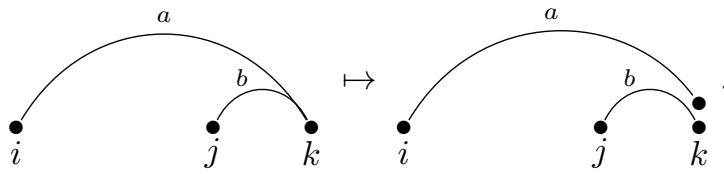

(TB) If $\left|R_{j k}\right|>1$, then

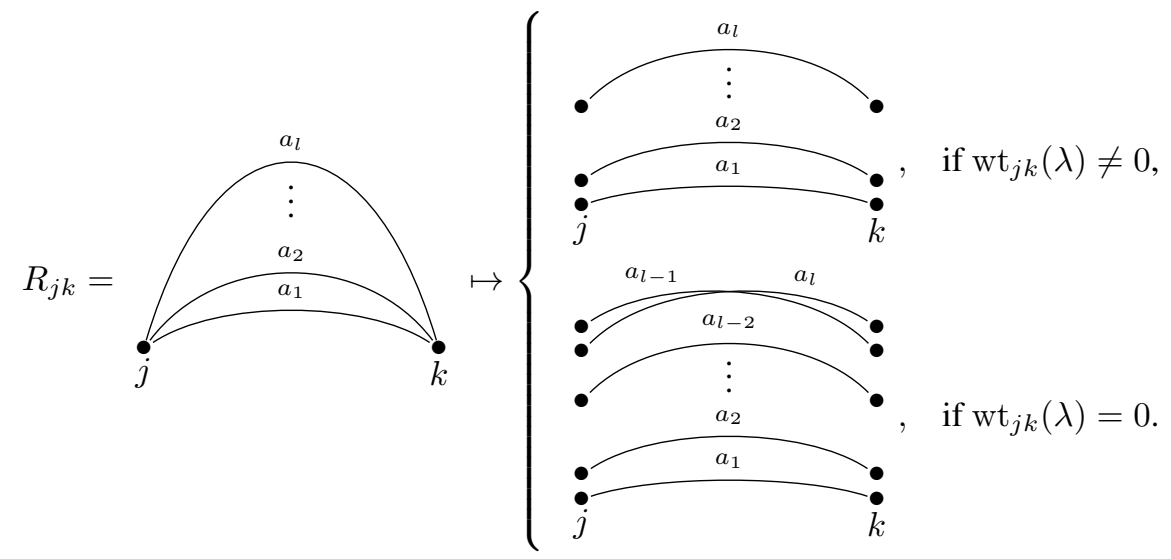


Example 3 For $q=3$,

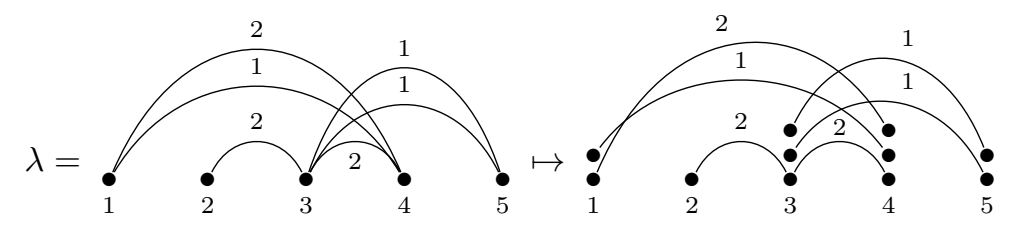

Define a labeling function $\Lambda_{K}: \lambda \rightarrow\{(\bullet, \bullet),(\circ, \bullet),(\bullet, \circ),(\circ, \circ)\}$, given by

$$
\Lambda_{K}(j \stackrel{b}{\circ} k)=\left(\Lambda_{K}^{L}(j \stackrel{b}{\sim} k), \Lambda_{K}^{R}(j \stackrel{b}{\sim} k)\right),
$$

where

$$
\begin{aligned}
& \Lambda_{K}^{L}(j \stackrel{b}{b} k)= \begin{cases}\circ & \text { if } j \notin K \text { or } j \stackrel{b}{\bullet} k \text { starts below an arc starting at } j, \\
\bullet & \text { otherwise. }\end{cases} \\
& \Lambda_{K}^{R}(j \stackrel{b}{\circ} k)= \begin{cases}\circ & \text { if } k \notin K \text { or } j \stackrel{b}{\circ} k \text { ends below an arc ending at } k, \\
\bullet, & \text { otherwise. }\end{cases}
\end{aligned}
$$

In the above example,

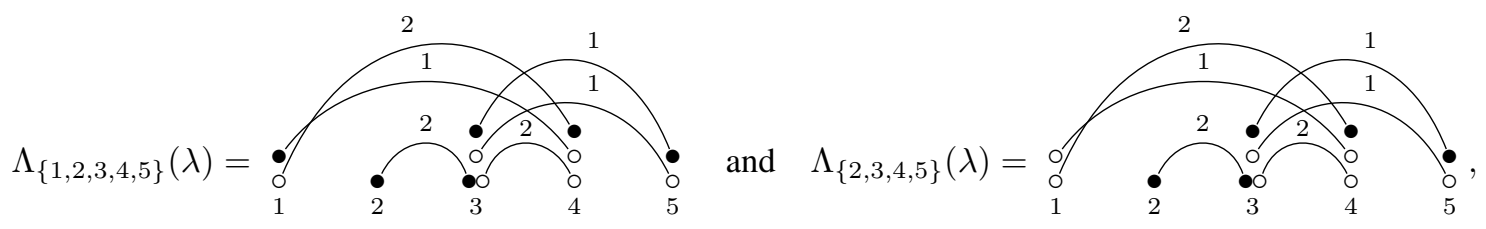

where we replace the endpoints of the arcs by their images under $\Lambda_{K}$.

Construct a bipartite graph $\Gamma_{K}(\lambda)$ given by vertices

$$
\begin{aligned}
& V_{\bullet}=\left\{j \stackrel{a}{\sim} k \in \lambda \mid \Lambda_{K}(j \stackrel{a}{\sim} k)=(\bullet, \bullet)\right\} \\
& V_{\circ}=\left\{j \stackrel{a}{\sim} k \in \lambda \mid \Lambda_{K}(j \stackrel{a}{\sim} k)=(\circ, \circ)\right\},
\end{aligned}
$$

and an edge from $i \stackrel{a}{\sim} l \in V_{\circ}$ to $j \stackrel{b}{口} k \in V_{\bullet}$ if $i<j<k<l$.

In our example,

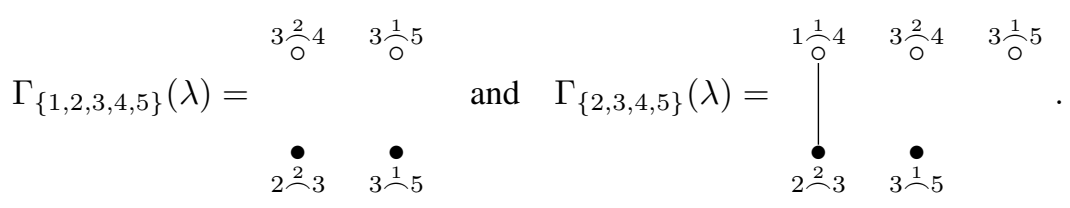

The main theorem of this section follows, and its proof can be found in Section 4.2

Theorem 4.1 Suppose $\lambda, \mu, \nu \in \mathcal{S}_{K}(q)$ with $K \subseteq \mathbb{Z}_{\geq 1}$ a finite subset. Then

$$
\left\langle\chi^{\lambda} \otimes \chi^{\mu}, \chi^{\nu}\right\rangle \neq 0
$$

if and only if $\Gamma_{K}(\lambda \cup \mu \cup \bar{\nu})$ has a complete matching from $V_{\bullet}$ to $V_{\circ}$. 


\subsection{Straightening rules}

Given $\lambda \in \mathcal{M}(q)$, this section describes "straightening" rules that allow us to create a sequence

$$
\lambda=\lambda^{(0)}, \lambda^{(1)}, \cdots, \lambda^{(\ell)}
$$

where at each stage we remove a conflict of type (CL), (CR), or (CB) until we arrive at $\lambda^{(\ell)} \in \mathcal{S}(q)$. Furthermore, there is an underlying sequence of pairs $\left(K^{(0)}, L^{(0)}\right),\left(K^{(1)}, L^{(1)}\right), \ldots,\left(K^{(\ell)}, L^{(\ell)}\right)$ of finite subsets such that $\left|K^{(i)}\right|=|K|$ and $\lambda^{(i)} \in \mathcal{M}_{K^{(i)} \cup L^{(i)}}(q)$. While the order in which one applies the straightening rules does matter in terms of which set partition one obtains, for our purposes in this paper (Theorem 4.2, below) the differences are irrelevant. The rules are as follows.

For $a, b \in \mathbb{F}_{q}^{\times}$, in moving from $\lambda^{(m-1)}$ to $\lambda^{(m)}$ we can

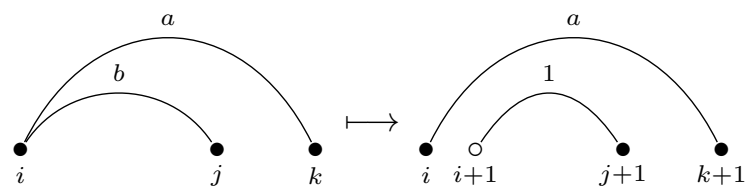

with

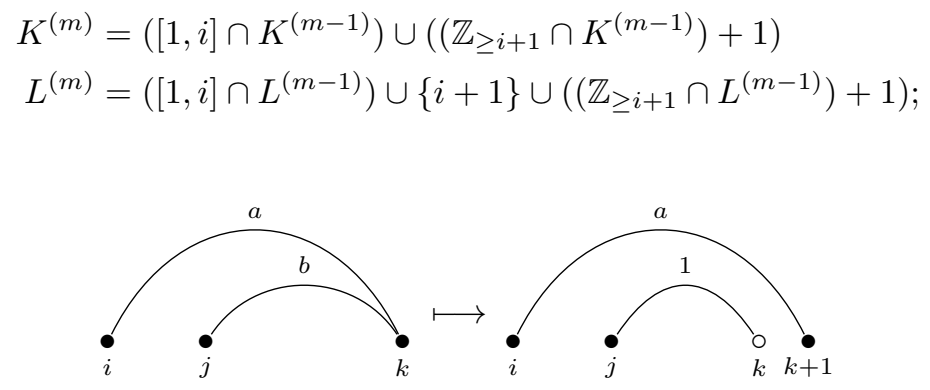

with

$$
\begin{aligned}
K^{(m)} & =\left([1, k-1] \cap K^{(m-1)}\right) \cup\left(\left(\mathbb{Z}_{\geq k} \cap K^{(m-1)}\right)+1\right), \\
L^{(m)} & =\left([1, k-1] \cap L^{(m-1)}\right) \cup\{k\} \cup\left(\left(\mathbb{Z}_{\geq k} \cap L^{(m-1)}\right)+1\right) ;
\end{aligned}
$$

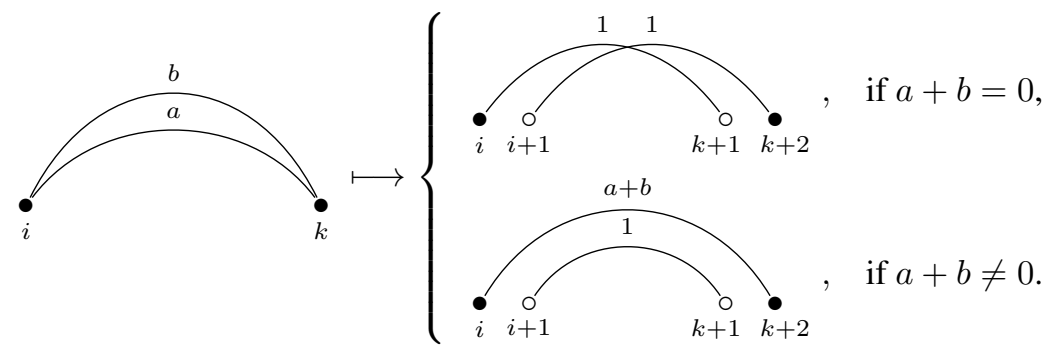


with

$$
\begin{aligned}
K^{(m)} & =\left([1, i] \cap K^{(m-1)}\right) \cup\left(\left([i+1, k-1] \cap K^{(m-1)}\right)+1\right) \cup\left(\left(\mathbb{Z}_{\geq k} \cap K^{(m-1)}\right)+2\right) \\
L^{(m)} & =\left([1, i] \cap L^{(m-1)}\right) \cup\{i+1\}\left(\left([i+1, k-1] \cap L^{(m-1)}\right)+1\right) \cup\{k+1\} \cup\left(\left(\mathbb{Z}_{\geq k} \cap L^{(m-1)}\right)+2\right) .
\end{aligned}
$$

In each case there are "new nodes" indicated by $\circ$ that push all the other node values to the right up (note, we view the $\bullet$-nodes as being the same though their number labels may change). In fact, $K^{(m)}$ is the set of original nodes (up to being pushed around) and $L^{(m)}$ is the set of nodes that were at some point o-nodes (see example after Theorem 4.2).

The following lemma states that these rules (SL), (SR) and (SB) do not fundamentally change the underlying character.

Lemma 4.1 Let $\lambda \in \mathcal{M}_{K}(q)$ and apply (SL), (SR) or $(S B)$ to obtain $\tilde{\lambda}=\lambda^{(1)} \in \mathcal{M}_{K^{(1)} \cup L^{(1)}}(q)$. Then as a character of $U_{K} \cong U_{K^{(1)}}$,

$$
\chi^{\lambda}=q^{-r_{K^{(1)}}^{K^{(1)} \cup L^{(1)}}(\tilde{\lambda})} \operatorname{Res}_{U_{K^{(1)}}^{U^{(1)} \cup L^{(1)}}}^{\left.U^{(\tilde{\lambda}}\right)} .
$$

By iterating Lemma 4.1 to remove all the conflicts of a multiset, we see that up to shifting of indices every tensor product is the same (up to a scalar multiple) as restriction from some supercharacter.

Theorem 4.2 Let $\lambda \in \mathcal{M}_{K}(q)$. Then there exists $\tilde{\lambda} \in \mathcal{S}_{K^{\prime} \cup L^{\prime}}(q)$ with $|K|=\left|K^{\prime}\right|$, such that

$$
\chi^{\lambda}=q^{-r_{K^{\prime}}^{K^{\prime} \cup L^{\prime}}(\tilde{\lambda})} \operatorname{Res}_{U_{K^{\prime}}}^{U_{K^{\prime}} \cup L^{\prime}}\left(\chi^{\tilde{\lambda}}\right) .
$$

Remark 3 Note that if we assume there are no (CN) conflicts, or $\lambda \in \mathcal{M}_{K}(q)$, then we may simplify the definition of the labeling function $\Lambda_{K}$ as follows. If $\lambda \in \mathcal{M}_{K}(q)$ and $\tilde{\lambda} \in \mathcal{S}_{K^{(\ell)} \cup L^{(\ell)}}(q)$ is obtained by applying $(S L),(S R)$ and $(S B)$, then

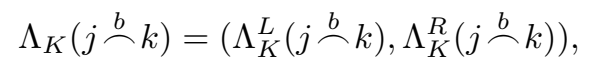

where

$$
\begin{aligned}
& \Lambda_{K}^{L}(j \stackrel{b}{\sim} k)= \begin{cases}\circ & \text { if } j \in L^{(\ell)}, \\
\bullet & \text { otherwise. }\end{cases} \\
& \Lambda_{K}^{R}(j \stackrel{b}{\stackrel{b}{S}} k)= \begin{cases}\circ & \text { if } k \in L^{(\ell)}, \\
\bullet & \text { otherwise. }\end{cases}
\end{aligned}
$$

Theorem 4.2 also allows us to extend Theorem 3.1 to the coefficient of arbitrary supercharacters.

Theorem 4.3 Suppose $\lambda \in \mathcal{S}_{L}(q)$ and $\mu \in \mathcal{S}_{K}(q)$ with $K \subseteq L \subseteq \mathbb{Z}_{\geq 1}$ finite sets. Then

$$
\left\langle\operatorname{Res}_{U_{K}}^{U_{L}}\left(\chi^{\lambda}\right), \chi^{\mu}\right\rangle \neq 0
$$

if and only if $\Gamma_{K}(\lambda \cup \bar{\mu})$ has a complete matching from $V_{\bullet}$ to $V_{\circ}$. 


\section{References}

[1] André, C. Basic characters of the unitriangular group, J. Algebra 175 (1995), 287-319.

[2] André, C. Irreducible characters of finite algebra groups, Matrices and group representations Coimbra, 1998 Textos Mat. Sér B 19 (1999), 65-80.

[3] André, C. The basic character table of the unitriangular group, J. Algebra 241 (2001), 437-471.

[4] André, C. Basic characters of the unitriangular group (for arbitrary primes), Proc. Amer. Math. Soc. 130 (2002), 1934-1954.

[5] André, C; Neto, A. Super-characters of finite unipotent groups of types $B_{n}, C_{n}$ and $D_{n}$, J. Algebra 305 (2006), 394-429.

[6] André, C; Neto, A. Supercharacters of the Sylow $p$-subgroups of the finite symplectic and orthogonal groups, Pacific J. Math. 239 (2009), 201-230.

[7] Arias-Castro, E; Diaconis, P; Stanley, R. A super-class walk on upper-triangular matrices, J. Algebra 278 (2004), 739-765.

[8] Diaconis, P; Isaacs, M. Supercharacters and superclasses for algebra groups, Trans. Amer. Math. Soc. 360 (2008), 2359-2392.

[9] Garsia, A; Remmel, J. Q-counting rook configurations and a formula of Frobenius, J. Combin. Theory Ser. A 41 (1986), 246-275.

[10] Halverson, T; Thiem, N. q-Partition algebra combinatorics. To appear in J. Combin. Theory Ser. A.

[11] Hendrickson, A. Supercharacter theories of finite cyclic groups. Unpublished Ph.D. Thesis, Department of Mathematics, University of Wisconsin, 2008.

[12] Higman, G. Enumerating p-groups I: Inequalities, Proc. London Math. Soc. 10 (1960), 24-30.

[13] Lewis, S. Restriction of Supercharacters in $U_{n}\left(\mathbb{F}_{2}\right)$. Honors thesis (2009), University of Colorado at Boulder.

[14] Marberg, E; Thiem, N. Superinduction for pattern groups, J. Algebra 321 (2009), 3681-3703.

[15] Otto, B. Two bounds for the nilpotence class of an algebra, 2009 preprint.

[16] Thiem, N. Branching rules in the ring of superclass functions of unipotent upper-triangular matrices. To appear in J. Algebraic Combin.

[17] Thiem, N; Venkateswaran, V. Restricting supercharacters of the finite group of unipotent uppertriangular matrices, Electron. J. Combin. 16(1) Research Paper 23 (2009), 32 pages.

[18] Yan, N. Representation theory of the finite unipotent linear groups, Unpublished Ph.D. Thesis, Department of Mathematics, University of Pennsylvania, 2001. 